\title{
Qu'est-ce qu'un verbe de déplacement ? : critères spatiaux pour une classification des verbes de déplacement intransitifs du français
}

\author{
Michel Aurnague \\ Cognition, Langues, Langage, Ergonomie (CLLE-ERSS, UMR 5263) \\ CNRS \& Université de Toulouse-Le Mirail \\ michel.aurnague@univ-tlse2.fr
}

\section{Introduction}

De nombreux travaux à l'interface entre syntaxe et sémantique se sont intéressés aux verbes de déplacement/mouvement dans le cadre de recherches générales sur l'articulation entre ces deux niveaux d'informations linguistiques (Jackendoff, 1983, 1990) ou de problématiques plus spécifiques mettant en jeu cette interface telle que l'opposition inaccusativité/inergativité (Levin \& Rappaport, 1992, 1995, Perlmutter, 1978) ou l'étude de phénomènes aspectuo-temporels (Krifka, 1992, 1995, Tenny, 1995).

Parallèlement, un grand nombre d'analyses se sont directement penchées sur la problématique de l'expression du déplacement dans la langue. Ces recherches ont pu être réalisées sur des langues particulières (pour s'en tenir au français on citera : Asher \& Sablayrolles, 1995, Boons, 1987, Borillo, 1998, Lamiroy, 1983, Laur, 1991, Sarda, 1999) ou avoir une portée plus large en tentant de mettre en évidence des différences typologiques remarquables du point de vue des moyens syntactico-sémantiques mis en œuvre pour décrire le déplacement (Bowerman et al., 1995, Grinevald, à paraître, Hickmann, 2006, Kopecka, 2006, Slobin, 2003, 2004, Stosic, 2002, Talmy, 1985, 2000).

Ces diverses investigations ont permis de faire d'importantes avancées dans la connaissance des éléments de sens que la langue retient pour décrire le déplacement de même que des catégories et structures syntaxiques à travers lesquelles ces éléments sont encodés. Une terminologie très riche a vu le jour au fil des études : mouvement dirigé, manière de se déplacer, changement de lieu, franchissement de frontière, direction, vecteur, trajet, trajectoire, source/départ (initial), but/arrivée (final), traversée (médian)... pour ne citer qu'une partie des nombreux vocables et expressions utilisés. Mais, comme il est fréquent dans le champ des sciences humaines - notamment en linguistique - des termes distincts désignent souvent des phénomènes identiques alors qu'inversement une dénomination unique peut, selon les auteurs, faire référence à des réalités différentes (voir, par exemple, la définition du terme «trajet " ('path') chez Jackendoff $(1983,1990)$ et Talmy (2000)). Une conséquence directe de cette variété d'approches et d'outils est que l'on aurait bien du mal à définir de façon convergente, sinon unitaire, ce qui constitue un procès (une "éventualité ») de déplacement dans la langue et la cognition.

L'objectif principal de cet article est précisément de mieux caractériser ce que la langue traite comme un procès/une éventualité de déplacement et cette question est essentiellement abordée à travers l'analyse des verbes intransitifs du français se référant à un déplacement «autonome » (quelques exemples de verbes transitifs sont cependant utilisés pour élargir le propos et la démonstration). Cette problématique générale présente deux versants principaux: quel(s) test(s) linguistique(s) permet(tent)-il(s), en français, de singulariser un déplacement?; quelles sont les propriétés sémantiques qui distinguent ce type d'éventualités d'autres catégories de procès (en particulier spatiaux) ?

La caractérisation des éventualités de déplacement que nous nous proposons de faire sera guidée par deux principes majeurs. Il s'agira, tout d'abord, de définir les éventualités concernées par leurs propriétés spatiales plutôt qu'à travers leurs caractéristiques aspectuo-temporelles (liées, le plus souvent, à l'aspect interne/lexical ou Aktionsart : Vendler, 1957, Vet, 1994, Vetters, 1996). Il est fréquent, par exemple, que, faute de critères spatiaux précis et opérationnels, le départ entre verbes exprimant la manière de se déplacer et verbes dénotant un véritable déplacement (déplacement au sens strict) se fasse à partir de propriétés aspectuelles (atélicité vs. télicité). Une mise en correspondance des propriétés spatiales et 
aspectuelles des procès n'est, bien sûr, pas exclue mais elle devra intervenir dans un second temps, sur la base d'une caractérisation spatiale claire.

Nous aurons pour deuxième exigence de veiller à ce que chaque notion (spatiale) utilisée soit définie le plus précisément possible, en s'assurant de sa cohérence avec les autres concepts qui interagissent avec elle dans la caractérisation d'un déplacement mais aussi de sa compatibilité avec des notions identiques ou équivalentes intervenant dans d'autres domaines de l'espace linguistique (ex : espace statique). Si, par exemple, l'opposition objet vs. lieu qui paraît sous-tendre le fonctionnement de certains marqueurs statiques indique qu'un tabouret ou un vélo sont catégorisés comme des objets ( ${ }^{*}$ Le chat est au tabouret ; * Max est au vélo), il sera difficile de postuler la présence d'un quelconque changement de lieu dans des énoncés tels que Le chat est allé sur le tabouret ou Max est descendu du vélo (voir plus loin une explicitation de ce point).

Nous n'aborderons pas, dans cet article, la distinction entre verbes de changement de posture (ex: s'asseoir, s'agenouiller, s'étirer, se lever, se recroqueviller, se (re)tourner) et verbes de changement d'emplacement (ex : avancer, foncer, glisser, grimper, marcher, patrouiller, nager, zigzaguer). Comme indiqué dans (Aurnague, à paraître), alors que les seconds dénotent un changement d'emplacement par rapport au cadre de référence terrestre (1), les premiers se réfèrent à des mouvements circonscrits au cadre que détermine l'entité-tout ce qui se manifeste, notamment, par la difficulté à les associer à des groupes prépositionnels en à travers (2). Bien qu'elle soit aujourd'hui assez largement admise, la notion de changement d'emplacement par rapport au cadre de référence terrestre n'est cependant pas simple à saisir et mobilise des paramètres complexes tels que le positionnement des surfaces de l'entité-cible (entité localisée) en contact avec le sol («surfaces portées »), la (les) position(s) et forme(s) canonique(s) de cette entité, sa manière habituelle de se mouvoir, etc., paramètres qui sont étroitement liés à la connaissance du monde et à la pragmatique.

$$
\begin{aligned}
& \text { Max a couru/rampé à travers le jardin } \\
& \text { ??Max s'est assis/étendu à travers le jardin }
\end{aligned}
$$

Nous examinons, dans la suite, les prédicats de déplacement habituellement caractérisés par les critères de télicité et/ou de changement de lieu (entre autres possibilités) et montrons que leur sémantisme fait appel aux notions spatiales de changement de relation locative élémentaire et de changement d'emplacement (section 2). Nous mettons ensuite en évidence la combinatoire complexe que dessinent ces deux concepts en observant, d'une part, les prédicats de changement d'emplacement susceptibles de participer à la description d'un changement de relation et d'emplacement (section 3.1) et, d'autre part, les différents types de procès auxquels réfèrent les verbes intransitifs (transitifs indirects) de changement de relation et d'emplacement.

\section{Déplacements « téliques » et notion de changement de relation (et d'emplacement)}

Cette section porte sur la classe de verbes de déplacement qui paraît, à bien des égards, la plus typique et connue (ex : aller + Prép, arriver, entrer, partir, se rendre, sortir). De nombreuses études distinguent ces prédicats de simples changements d'emplacement (en particulier, de verbes indiquant la manière du déplacement) en remarquant que, contrairement à ces derniers, ils dénotent des procès téliques (accomplissements ou achèvements). Comme il a pu être mis en évidence (Aurnague, 2000, Aurnague \& Stosic, 2002, Stosic, 2002, 2007), ces deux classes de verbes se comportent de manière différente en présence d'un groupe prépositionnel en par. Alors que les énoncés intégrant un changement d'emplacement semblent difficilement acceptables pour de nombreux locuteurs actuels ou sont, au mieux, interprétés au moyen d'un emploi «imprécis» de par $(3-4)^{2}$, les verbes examinés ici autorisent une interprétation de type «trajet» de cette préposition (5-6), dans laquelle l'entité dénotée par son objet nominal est supposée connecter plusieurs éléments de la trajectoire ou du parcours de la cible.

?Max a couru/marché par (tout) le bois/les coteaux

(4) (?)Max a déambulélerré par (toute) la ville/les rues piétonnes 
Ce type de description (avec GP en par) illustre les propriétés spatiales des verbes considérés et souligne la nécessité d'une caractérisation qui ne soit pas uniquement fondée sur l'aspect interne ou Aktionsart. Une identification spatiale de ces lexèmes est parfois proposée mais il est fréquent que les propriétés mises en avant soient à peine (ou pas) définies et jouent le rôle de simples étiquettes : le concept de « déplacement dirigé » (Lamiroy, 1983, Levin, 1993, Levin \& Rappaport, 1992, 1995) en est un bon exemple dans la mesure où la notion de direction ne garantit pas l'appartenance à la classe de prédicats envisagée ici (certains changements d'emplacement font, en effet, appel à la directionnalité : cf. (Aurnague, à paraître) et section 3.1). D'autres propositions recourent à des définitions spatiales plus précises mais qui ne sont pas, pour autant, adéquates. C'est le cas des nombreuses approches, parmi lesquelles (Jackendoff, 1983, 1990), qui associent systématiquement au site (entité de référence ou localisatrice) d'une description statique ou dynamique une portion d'espace/région - généralement qualifiée de lieu - dans laquelle la cible est localisée. (Asher \& Sablayrolles, 1995) considèrent que les verbes de déplacement téliques analysés expriment des changements de lieux et étendent l'approche en termes de régions en affectant à chaque site un ensemble de zones qui permettent d'opérer des distinctions plus fines parmi ces prédicats (contraintes sur la « source » et le «but » du déplacement).

Plusieurs critiques peuvent être adressées aux propositions faisant appel à des régions/lieux. Ainsi, (a) elles ne tiennent pas compte du contenu fonctionnel des marqueurs spatiaux (ex : contenance, support) dont le rôle est pourtant essentiel (Vandeloise, 1986, 2001) ${ }^{3}$ et (b) tendent à réduire les aspects géométriques de la localisation à la seule relation d'inclusion. Par ailleurs, (c) la véritable nature des entités dont on postule l'existence (régions/lieux) et leur ancrage linguistique sont rarement discutés aboutissant, par exemple, à l'introduction de très nombreux éléments (cf. zones de Asher \& Sablayrolles, 1995) ou à des caractérisations qui rendent incompatibles l'expression de l'espace statique et dynamique. A ce propos, et comme évoqué dans l'introduction, la règle d'usage proposée dans (Vandeloise, 1988) pour saisir les emplois (statiques) localisateurs de la préposition à paraît sélectionner des entités-sites correspondant à des « lieux spécifiés » (Aurnague, 1996, 2004). Un lieu est ici défini comme une entité (matérielle) fixe/stable dans un cadre de référence donné qui, en outre, détermine une portion d'espace (Aurnague et al., 2007). Contrairement aux entités géographiques ou aux entités mixtes/habitations (ex : Le chat est au grenier; Max est au hangar), un tabouret, un vélo (voir introduction), un seau ou un tapis ne remplissent pas ces conditions (absence de fixité et/ou de portion d'espace) et fonctionnent en tant qu'objets dans les descriptions statiques : La mouche est dans le/*au seau; L'enfant est sur le/*au tapis. Il n'y a, de fait, aucune raison de penser que ces entités acquièrent le statut de lieu dans des énoncés dynamiques (ex: La mouche est entrée dans le seau; L'enfant est venu sur le tapis), ce qui exclut une caractérisation des verbes ou locutions verbales correspondants en termes de changement de lieu.

Dans sa classification syntactico-sémantique des verbes de déplacement du français, J.P. Boons (1987) a mis en évidence une notion très intéressante et opératoire, celle de changement de relation locative élémentaire, malheureusement peu utilisée depuis (mis à part dans Muller \& Sarda, 1998). Cette notion permet à l'auteur de distinguer les verbes « initiaux ou finaux unipolaires » (également qualifiés de verbes « causatifs » de déplacement; ex : adosser, défricher, dévisser) des prédicats qui constituent, selon lui, de véritables déplacements (ex : chasser, enfourner, hisser). Ainsi, alors qu'un prédicat du type adosser ne repose pas sur la notion de changement de relation locative élémentaire (on peut adosser à un mur une armoire qui était déjà en contact avec lui : la relation niée puis affirmée - être adossé à - n'est pas une relation locative élémentaire), un verbe comme enfourner met bien en jeu une telle relation puisque la négation et l'affirmation de la relation spatiale être dans sous-tend sa sémantique. On remarque assez rapidement que le concept de changement de relation locative élémentaire permet d'écarter de la classe de prédicats qui nous occupe les procès de changement d'emplacement (ex : marcher/se promener (dans le parc) ne met en jeu aucun changement de relation par rapport à un site éventuel). Au contraire, un verbe tel que entrer implique bien un changement de relation locative élémentaire. Bien qu'elle constitue une avancée significative dans la perspective d'une classification et d'une analyse des verbes qui soient linguistiquement motivées, cette notion s'avère ne pas être suffisante. En raison de leur contenu 
fonctionnel, certaines relations locatives élémentaires peuvent, en effet, être niées puis affirmées (ou l'inverse) sans impliquer un réel déplacement. Ceci se produit, par exemple, dans la phrase (7) (emploi de $a \grave{a}$ de type «routine sociale »: Vandeloise, 1988) qui peut parfaitement se référer à une situation dans laquelle Max, assis sur un fauteuil/tabouret pivotant, s'installe alternativement à son bureau ou à son piano sans, pour autant se déplacer. De la même façon (8), le fait que Max lâche le plat qu'il maintenait en léger contact avec la table (la table supporte désormais le plat) induit un changement de relation locative élémentaire (sur) qui ne s'accompagne pas d'un déplacement. ${ }^{4}$ Ces observations n'invalident pas totalement la proposition de J.P. Boons: elles suggèrent simplement de combiner les notions de changement de relation ${ }^{5}$ et de changement d'emplacement pour caractériser les prédicats téliques de déplacement envisagés ici, ce que nous nous proposons de faire.

\section{Max s'est installé à son piano/sa table de travail} Max a mis le plat sur la table

L'association de ces deux notions aboutit à une riche combinatoire faisant appel à deux référents distincts : le cadre de référence terrestre par rapport auquel est évalué le changement d'emplacement (cf. introduction) et l'entité-site (explicitement mentionnée dans la description ou sous-entendue) permettant d'établir l'existence d'un possible changement de relation. On a souligné, plus haut, que les changements d'emplacement n'impliquent, en eux-mêmes, aucun changement de relation locative élémentaire. Nous venons de voir, par ailleurs, que des changements de relation portant sur des propriétés fonctionnelles peuvent ne pas s'accompagner de changement d'emplacement. Un examen plus approfondi indique que certains changements de relation locative élémentaire mettant à la fois en jeu contenu fonctionnel et géométrique ne semblent pas constituer des changements d'emplacement. Ceci transparait, en particulier, dans l'interprétation des verbes se poser et (sans doute aussi) se percher qui, appliqués à des oiseaux, supposent généralement l'introduction conjointe du contact et du support. La phrase (9), par exemple, révèle qu'en dépit du changement de relation locative élémentaire (propriétés géométriques et fonctionnelles de sur), le procès décrit n'est pas véritablement catégorisé comme un changement de relation et d'emplacement par la langue et la cognition (l'intégration d'un GP en par est problématique). ${ }^{6}$

\section{?(?) L'oiseau s'est posé/perché sur la maison par le jardin}

(10) L'oiseau est entré dans la maison par le jardin

Il semble que, pour être davantage acceptable, (9) nécessite un contexte particulier dans lequel, par exemple, l'accessibilité à la maison soit rendue difficile. L'action de se poser serait alors réinterprétée de façon à intégrer l' " approche » du site et ajouter un changement d'emplacement au seul changement de relation. Dans (Aurnague \& Stosic, 2002) et (Aurnague, 2004), nous avons déjà noté que des verbes comme atterrir et décoller utilisés dans le domaine aéronautique sont susceptibles de dénoter un changement d'emplacement précédant ou suivant le changement de relation locative élémentaire.

L'effet conjoint des changements de relation et des changements d'emplacement dans l'élaboration de descriptions dynamiques autorisant le recours à un GP en par se manifeste aussi par le fait que de nombreux prédicats de (simple) changement de relation peuvent apparaître avec de tels GP lorsqu'ils sont associés au verbe aller au sein d'une construction infinitive directe (11). Ces constructions, qui ont été l'objet d'une étude poussée dans (Lamiroy, 1983), font, par elles-mêmes, appel à un changement de relation et d'emplacement final et autorisent, par conséquent, l'ajout d'un GP en par. Un aspect extrêmement intéressant de ces structures est que le verbe de déplacement de la proposition principale n'est compatible avec un prédicat de changement de relation dans l'infinitive directe que si ce dernier n'exprime pas également un changement d'emplacement (cf. 11-12 ainsi que *Max est parti se rendre à Paris).

(11) L'oiseau est allé se poser/percher sur la maison (par le jardin)

(12) *L'oiseau est allé entrer dans la maison (par le jardin)

Les notions de changement de relation et de changement d'emplacement permettent donc de délimiter plus précisément les verbes téliques souvent considérés comme des déplacements au sens strict, leur association à un GP en par et leur intégration dans une proposition infinitive directe dessinant, comme on 
peut le constater, des distributions complémentaires $(9-10 ; 11-12)$. Des phénomènes plus subtils (que nous n'aborderons pas ici) peuvent être mis en évidence: ainsi, lorsqu'ils sont présents dans le sémantisme verbal, changement de relation et/ou changement d'emplacement sont parfois relégués à une place secondaire par d'autres aspects du procès - en particulier l'affectation du site - qui paraissent davantage soulignés (ex : emplois transitifs vs. intransitifs de $\left(s^{\prime}\right)$ infiltrer/pénétrer) (Aurnague, à paraître).

\section{Changement de relation et changement d'emplacement : une combinatoire complexe}

Cette section examine plus en détail les interactions entre changement de relation et changement d'emplacement en considérant, tout d'abord, les prédicats de changement d'emplacement pouvant apparaître dans des descriptions de changement de relation et d'emplacement puis en distinguant différents types de procès au sein des verbes intransitifs de changement de relation et d'emplacement.

\subsection{Quand un changement de relation s'associe à un changement d'emplacement : la notion de «tendancialité »}

La combinaison des verbes de changement d'emplacement et des GP spatiaux ( « finaux ») a fait l'objet de très nombreuses études en raison des propriétés particulières que présentent ces constructions du point de vue aspectuel (passage à une description télique : Krifka, 1995, Tenny, 1995) comme du point de vue de l'opposition inaccusatif/inergatif (Levin \& Rappaport, 1992, 1995). Sur un plan typologique (Jackendoff, 1990, Slobin, 2003, 2004, Talmy, 2000), il a été postulé que les langues qui, comme l'anglais, codent la composante "Trajet» ('Path') d'un déplacement au moyen de satellites ('satellite-framed languages') plutôt qu'à travers leur verbe avaient une propension particulière à utiliser ce type de descriptions (ex : Max walked into the meadow 'Max est allé dans le pré en marchant'). Les idiomes qui, comme le français, expriment le Trajet via le verbe ('verb-framed languages') seraient, au contraire, beaucoup plus rétifs à décrire un changement de relation et d'emplacement avec cette construction (Max a marché dans le pré peut uniquement dénoter un changement d'emplacement). La plupart des travaux soutiennent que ce type de description est très rare dans les langues considérées et, sauf exception (Fong \& Poulain, 1998), cette affirmation a peu été remise en cause. Si l'expression d'un changement de relation et d'emplacement au moyen d'un verbe de changement d'emplacement et d'un GP est, sans conteste, davantage contrainte en français qu'en anglais, elle est, néanmoins, beaucoup plus productive qu'on a bien voulu le dire. C'est ce que nous montrons, en mettant en évidence les caractéristiques sémantiques des verbes de changement d'emplacement autorisant ce type de construction et d'interprétation.

La première catégorie de verbes de changement d'emplacement susceptibles de s'associer à un GP pour décrire un changement de relation et d'emplacement est constituée de prédicats soulignant la vitesse du déplacement. C'est le cas de courir, galoper (et peut-être trotter) (13) ainsi que de foncer ou filer (14). Comme il est assez largement admis, les deux interprétations disponibles se distinguent par la fonction syntaxique du GP qui peut avoir le statut de simple modifieur (adjoint de la phrase, du VP...; changement d'emplacement) ou constituer un véritable complément du verbe (changement de relation et d'emplacement).

(13) Max a couru/galopé/(?)trotté à la cuisine

(14) Max a foncé/filé dans le couloir

On trouve, dans un second groupe, les verbes ramper et se traîner indiquant (pour les humains tout au moins) que le changement d'emplacement nécessite un effort particulier. Celui-ci vise à surmonter certaines « forces » - externes à la cible ou liées à elles - qui contrarient la réalisation du déplacement ou le rendent plus difficile. Comme auparavant, ces verbes associés à un GP approprié (« final») donnent lieu à une double interprétation dont l'une met en jeu un changement de relation et d'emplacement :

(15) Max a rampé sur la terrasse

(16) Max s'est traîné au salon 
Les prédicats directionnels de changement d'emplacement (ex : avancer, dégringoler, descendre, dévaler, grimper, se hisser, monter, reculer) forment le troisième groupe de verbes pouvant, en association avec un GP, faire référence à un changement de relation et d'emplacement. Nous postulons que ces prédicats dénotent d'abord, et de façon primitive, des changements d'emplacement (déplacement selon une direction particulière: sur ce point, nous rejoignons Sarda, 1999) ${ }^{7}$ et leur intégration à un énoncé comportant un GP adéquat permet, ici encore, l'émergence des deux interprétations considérées :

(17) Max a avancé dans le couloir

(18) Le chamois a dévalélest descendu dans le ravin

L'entrânement par une force constitue la quatrième et dernière propriété que nous avons pu mettre en évidence parmi les facteurs favorisant la transformation d'un changement d'emplacement en changement de relation et d'emplacement. Ce concept sous-tend le sémantisme de verbes tels que couler, dégouliner, déraper, glisser ou rouler qui supposent qu'une ou plusieurs forces, essentiellement extérieures à la cible, provoquent le déplacement. Ces forces externes (gravité, impact/impulsion, obstacle, etc.), éventuellement associées à des propriétés internes de la cible statique ou mobile (forme ou consistance, élan, etc.) viennent rompre son " équilibre » initial et l'entraînent dans un déplacement qui n'est pas de son fait. Il convient de remarquer que la notion d'équilibre d'une cible statique ou mobile utilisée ici et, plus généralement, le rôle assigné aux forces vont clairement au-delà de la théorie de la « dynamique des forces » de Talmy (2000), raison pour laquelle nous ne recourons pas à la terminologie correspondante (ex : agoniste vs. antagoniste). Quoi qu'il en soit, et comme auparavant, un déplacement causé par une force extérieure à la cible rend possible une interprétation basée sur un changement de relation et d'emplacement pour peu que le prédicat soit associé à un GP « final » approprié :

(19) La peinture a coulé/dégouliné sur le carrelage

(20) Max a glisséldérapé dans le ravin

Comme on peut le constater, et en accord avec d'autres études récentes (Kopecka, à paraitre), les verbes de changement d'emplacement du français pouvant entrer dans des énoncés dénotant des changements de relation et d'emplacement sont nettement plus nombreux qu'il n'est généralement admis. Le contraste qui, selon nous, s'établit entre langues de type « verb-framed » ou « satellite-framed » ne réside donc pas dans l'absence (ou quasi-absence) vs. présence de telles constructions mais dans leur apparition contrainte vs. pas ou peu contrainte. Les propriétés sémantiques qui, en français, conditionnent ce type d'emploi vitesse du déplacement, opposition (intentionnelle) à une force, direction (déplacement linéaire orienté) et entraînement par une force - suggèrent toutes la visée ou la «tendancialité » du déplacement, dans la mesure où le changement d'emplacement a la potentialité de «tendre » vers un site ou un but : nous considérerons que ces propriétés s'organisent en une ressemblance de famille sous-tendant la notion de tendancialité.

Ainsi qu'on a pu l'entrevoir au fil des exemples - et ceci est habituel des ressemblances de famille -, il n'est pas rare qu'un prédicat intègre plusieurs des propriétés mentionnées. Certains verbes indiquant la direction du déplacement peuvent, par exemple, associer cet élément à la notion d'opposition à une force (ex : grimper, se hisser) ou à celle de vitesse (ex : débouler, dégringoler, dévaler). ${ }^{8}$ Le verbe filer fournit, à lui seul, une belle illustration de ce phénomène dans la mesure où l'une de ses acceptions réfère à un parcours linéaire orienté ainsi qu'à l'entraînement par une force (nouveau Petit Robert: "(Prendre la forme d'un fil). Couler lentement sans que les gouttes se séparent») alors qu'une autre combine la direction à la vitesse (« Aller droit devant soi, en ligne droite ; aller vite »).

\subsection{Changement de relation, changement d'emplacement et catégorisation des verbes intransitifs}

Nous examinons maintenant les principaux verbes intransitifs de changement de relation et d'emplacement du français que nous classifions en fonction des rapports qui s'établissent entre ces deux éléments de leur sémantisme. Nous avons retenu, pour cette analyse, les lexèmes les plus représentatifs ${ }^{9}$ des listes établies dans (Laur, 1991) à partir de (Boons et al., 1976, Gross, 1975, Guillet, 1990). Les 
verbes ou locutions verbales se référant uniquement à un changement d'emplacement (ex : prédicats dénotant la manière du déplacement; cf. introduction) ou à un changement de relation (ex : prédicats basés sur la relation de contact/support; cf. section 2) ont, bien sûr, été expurgés de ces inventaires. La notion de polarité d'un déplacement, déjà évoquée, joue un rôle important dans cette classification (tout comme dans les travaux cités ci-dessus) et fait l'objet d'une définition précise fondée sur le concept de changement de relation. Un déplacement (au sens strict, c'est-à-dire un changement de relation et d'emplacement) est dit de polarité «initiale » si le changement de relation locative élémentaire qui le sous-tend repose sur l'affirmation de cette relation puis sur sa négation (l'information "positive » est initiale : $\mathrm{r} \ldots \triangleright \neg \mathrm{r}) .^{10}$ Cette polarité est, au contraire, «finale» lorsque l'affirmation de la relation élémentaire succède à sa négation (information positive finale : $\neg \mathrm{r} \cdots \triangleright \mathrm{r}$ ). Selon le même principe, un changement de relation «médian» se caractérisera par une information positive (affirmation de la relation) précédée et suivie de la négation de la relation sous-jacente $(\neg \mathrm{r} \ldots \triangleright \mathrm{r} \ldots \triangleright \neg \mathrm{r})$. Soulignons, cependant, qu'en dehors de locutions verbales du type couper par ou passer par, très peu de verbes intransitifs du français dénotant des changements de relation et d'emplacement peuvent être qualifiés de médians. Les classes de procès de changement de relation et d'emplacement dégagées sont regroupées dans le Tableau 1. Nous commentons quatre d'entre elles en raison de l'intérêt particulier qu'elles présentent.

La catégorie des prédicats se référant à un changement de relation initial indépendant est principalement représentée par le verbe partir et, dans une moindre mesure, par $s$ 'en aller (ainsi que par certaines variantes populaires comme se barrer). La relation locative élémentaire (affirmée puis niée) sous-tendant ces prédicats n'est pas réductible aux seules configurations d'inclusion/contenance (Max peut parfaitement partir de chez lui alors qu'il se trouve sur la terrasse ou devant la porte de sa maison) et semble souvent mieux correspondre aux situations que dénote la préposition à dans son emploi statique localisateur (Vandeloise, 1988). La seconde caractéristique de ces verbes réside dans le fait qu'ils se limitent à la description d'un changement de relation (et d'emplacement) initial et n'incluent pas dans leur sémantisme le déplacement "subséquent » auquel réfère le GP final qui leur est éventuellement associé (cf. Tableau 1; le déplacement subséquent correspond à l'événement e'). La phrase Max est parti à l'université à 8 heures est, de ce point de vue, spatio-temporellement équivalente à la description en discours Max est parti à 8 heures. Il allait à l'université (relation d'" Arrière-Plan»). Outre la modification par un adverbial en en, divers énoncés imperfectifs confirment que les procès concernés sont centrés sur le seul changement de relation et d'emplacement. C'est le cas, notamment, des descriptions au présent - Max part à l'université n'est en principe utilisable qu'au moment du changement de relation (et d'emplacement) - et des phrases à l'imparfait comportant une subordonnée temporelle (21). ${ }^{11}$ L'éventualité introduite par la proposition subordonnée de (21) est ainsi contemporaine du changement de relation initial plutôt que du déplacement qui lui fait possiblement suite (à moins de réinterpréter/accommoder la phrase en substituant aller/se rendre à à partir à).

\section{Max partait à l'université lorsque la pluie s'est mise à tomber}

S'échapper, s'enfuir, se sauver - de même que les verbes plus populaires se carapater, se cavaler, se tailler, se trotter, etc. - introduisent un changement de relation initial étendu et forment, sur la base de ce critère, une seconde catégorie de prédicats. Comme pour les verbes du groupe précédent, le procès décrit est réalisé dès lors que le changement de relation a eu lieu (Pollux le chien s'est échappé du restaurant est vraie aussitôt que la cible a quitté le site) et leur sémantisme paraît, ici encore, centré sur ce changement de relation (et d'emplacement) initial. Cependant, d'autres tests linguistiques mettant en jeu des énoncés dans lesquels les verbes considérés sont associés à un GP final semblent pointer la possibilité pour ces constructions de se référer à un déplacement subséquent au changement de relation et d'emplacement initial : ajout d'un adverbial en en, descriptions au présent coïncidant avec le changement de relation ou postérieures à celui-ci, phrases à l'imparfait avec subordonnée temporelle (22: l'événement de la subordonnée peut se produire durant le déplacement subséquent).

$$
\text { Max s'enfuyait/se sauvait au village lorsque la pluie s'est mise à tomber }
$$


Une manifestation supplémentaire du comportement ambivalent des changements de relation initiaux étendus - centrage sur le changement de relation et d'emplacement initial mais possibilité de décrire, sous certaines conditions, un déplacement postérieur - est que l'interprétation des énoncés perfectifs niant la réalisation d'un déplacement subséquent peut apparaître moins immédiate pour ces verbes qu'elle ne l'est pour les changements de relation initiaux indépendants : ?Max s'est enfui/sauvé au village mais il n'y est jamais arrivé vs. Max est parti à l'université mais il n'y est jamais arrivé. ${ }^{12}$

Les prédicats de cette seconde classe se distinguent des changements de relation initiaux indépendants par le fait qu'ils soulignent la vitesse du déplacement effectué et indiquent, en même temps, la volonté de la cible de se soustraire au contrôle exercé sur elle par le site. Ce sont, très certainement, ces éléments - et, en particulier, la vitesse - qui permettent aux verbes examinés de décrire parfois un changement d'emplacement postérieur au changement de relation et d'emplacement initial. En présence d'un GP (final), et conformément à ce qui a été mis en évidence dans la section 3.1 (tendancialité), un changement de relation final vient s'ajouter à ce changement d'emplacement. La référence à un déplacement subséquent n'est donc pas, selon nous, un élément constitutif du sémantisme des verbes de cette classe mais doit plutôt être vue comme un «effet de bord » imputable à la présence, dans ce sémantisme, de propriétés bien spécifiques.

Tableau 1 Catégories de verbes intransitifs de changement de relation et d'emplacement

\begin{tabular}{|c|c|}
\hline fs initiaux & itits tinaux \\
\hline $\begin{array}{l}\text { Changement de relation initial indépendant : } \\
\text { partir } \\
{[]} \\
\text { e } \\
\begin{array}{ll}\mathrm{r}(\mathrm{c}, \mathrm{s}) \ldots \triangleright \neg \mathrm{r}(\mathrm{c}, \mathrm{s}) & \mathrm{e} \\
+\mathrm{ch}-\mathrm{empl} & \text { ch-empl } \\
+ \text { ch-rel }\end{array}\end{array}$ & 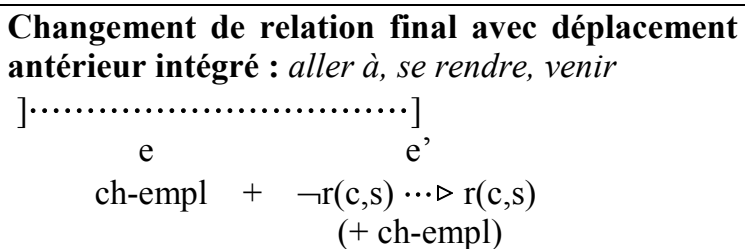 \\
\hline 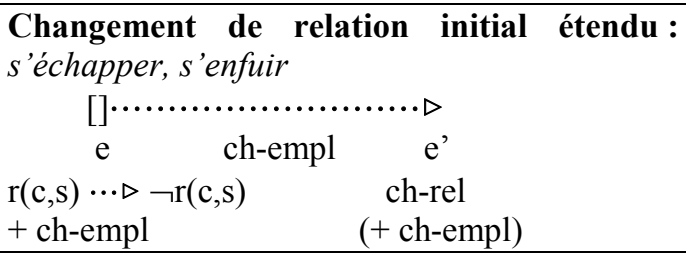 & $\begin{array}{l}\text { Changement de relation final avec déplacement } \\
\text { antérieur présupposé : arriver, parvenir } \\
\text { ].......................... } \\
\begin{array}{r}\mathrm{e} \\
\text { / ch-empl } \leftrightarrow / \quad \neg \mathrm{r}(\mathrm{c}, \mathrm{s}) \cdots \triangleright \mathrm{r}(\mathrm{c}, \mathrm{s}) \\
+\mathrm{ch}-\mathrm{empl}\end{array}\end{array}$ \\
\hline 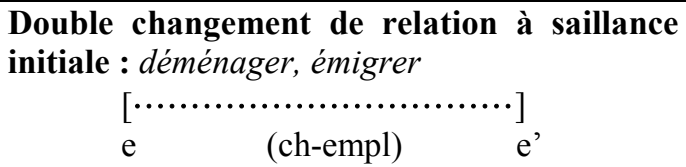 & $\begin{array}{l}\text { Double changement de relation à saillance } \\
\text { finale : } \text { immigrer } \\
\qquad \ldots \ldots \ldots \ldots \ldots \ldots \ldots \ldots \ldots \ldots] \\
\mathrm{e} \quad(\mathrm{ch}-\mathrm{empl})\end{array}$ \\
\hline $\begin{array}{l}\underline{\mathrm{r}(\mathrm{c}, \mathrm{s} 1) \cdots \triangleright \neg \mathrm{r}(\mathrm{c}, \mathrm{s} 1)}+\quad \begin{array}{l}\neg \mathrm{r}(\mathrm{c}, \mathrm{s} 2) \cdots \triangleright \mathrm{r}(\mathrm{c}, \mathrm{s} 2) \\
+\mathrm{ch}-\mathrm{empl}\end{array} \\
\end{array}$ & $\begin{array}{l}\mathrm{r}(\mathrm{c}, \mathrm{s} 1) \cdots \triangleright \neg \mathrm{r}(\mathrm{c}, \mathrm{s} 1)+\frac{\neg \mathrm{r}(\mathrm{c}, \mathrm{s} 2) \cdots \triangleright \mathrm{r}(\mathrm{c}, \mathrm{s} 2)}{+ \text { ch-empl }} \\
+ \text { ch-empl }\end{array}$ \\
\hline $\begin{array}{cc}\text { Changement de } & \text { relation initial de type } \\
\text { incl./cont. : sortir } & \text { représ. alternative } \\
{[]} & {[]} \\
\mathrm{e} & \mathrm{e} \\
\mathrm{r}(\mathrm{c}, \mathrm{s}) \ldots \triangleright \neg \mathrm{r}(\mathrm{c}, \mathrm{s}) & \mathrm{r}(\mathrm{c}, \mathrm{s}) \ldots \triangleright \mathrm{r}^{\prime}(\mathrm{c}, \mathrm{s}) \\
+ \text { ch-empl } & +\mathrm{ch}-\mathrm{empl} \\
\mathrm{r}=\text { incl./cont. } & \end{array}$ & 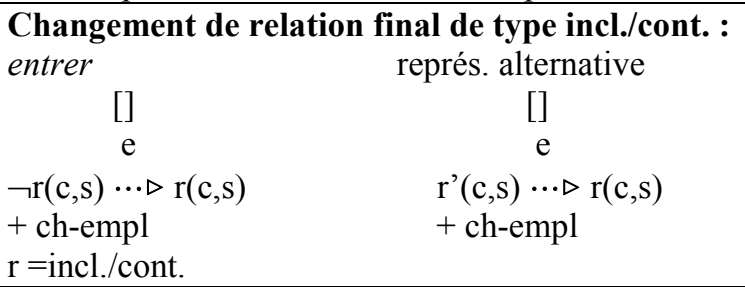 \\
\hline
\end{tabular}

c : cible, s : site; les crochets délimitent le contenu sémantique des verbes ; le soulignement indique le caractère saillant du changement de relation et d'emplacement correspondant

La troisième classe de prédicats que nous souhaitons mettre en évidence regroupe des verbes comme aller à (et, plus généralement, aller + Prép), se rendre ou venir - ainsi que certaines formes plus populaires : $s$ 'abouler, s'amener, rappliquer, etc. - fondés sur un changement de relation final avec déplacement antérieur intégré. Nous considérons, en effet, que le contenu sémantique de ces prédicats comprend un changement d'emplacement suivi d'un changement de relation (et, le cas échéant, d'emplacement) final (cf. Tableau 1). C'est, le plus souvent, l'aspect perfectif du temps utilisé (ex : passé composé) qui permet 
d'assigner une borne temporelle initiale au procès (et, indirectement, un changement de relation locative initial).$^{13}$ Certaines constructions associant un verbe de changement d'emplacement (indiquant la vitesse ou l'opposition à une force) et un GP et susceptibles de dénoter un changement de relation et d'emplacement (cf. section 3.1) sont, d'un de point de vue sémantique, très proches des verbes de cette catégorie et l'on a, de fait, probablement affaire au type de procès le plus répandu parmi ceux présentés dans ce travail.

Arriver, aboutir et parvenir appartiennent à la même catégorie de verbes, leur contenu sémantique consistant en un changement de relation final avec déplacement antérieur présupposé. Si ces prédicats dénotent un changement de relation (et d'emplacement) final sans désigner, pour autant, un changement d'emplacement antérieur, leur sémantisme «présuppose» bien l'existence d'un tel déplacement. ${ }^{14} \mathrm{Ce}$ changement d'emplacement présupposé explique la propriété aspectuelle bien connue de ces verbes, à savoir leur faculté de fonctionner comme des «achèvements » (possibilité d'ajout d'un adverbial en $a$ ) aussi bien que comme des «accomplissements 》 (adverbial en en) (23). Cette propriété présente d'ailleurs un pendant spatial - plus basique et, à notre connaissance, rarement relevé (Aurnague, 2000) - puisque la préposition par peut aussi bien introduire une entité ( site secondaire ») directement connectée au site final (et donc impliquée dans le changement de relation final) qu'une entité plus distante de ce site final, située sur la trajectoire antérieure de la cible (changement d'emplacement) (24).

\section{(23) Max est arrivé à l'université à 10 heures/en 10 minutes}

(24) Les réfugiés sont parvenus en France par l'Aragon/le Portugal

Les quatre classes de verbes restantes - dont nous n'approfondirons pas la présentation - comprennent, d'une part, des doubles changements de relation à saillance initiale ou finale, dont le sémantisme fait appel à une forme de «typage » des sites mis en jeu (ex : déménager, émigrer, immigrer) et, d'autre part, des changements de relation initiaux ou finaux basés sur l'inclusion/contenance (préposition dans ; ex : sortir, entrer, pénétrer).

En l'état actuel, la catégorisation obtenue (et, plus particulièrement, les quatre classes de verbes dont nous avons décrit les propriétés : moitié supérieure du Tableau 1) révèle une dissymétrie importante entre procès initiaux et finaux puisque les prédicats de changement de relation initiaux n'indiquent généralement pas, dans leur sémantique, l'existence d'un changement d'emplacement subséquent alors que les changements de relation finaux peuvent intégrer un changement d'emplacement antérieur ou le présupposer. Plus généralement, et mis à part les prédicats recourant au typage ou, dans une moindre mesure, à la relation d'inclusion/contenance, on constate que le contenu sémantique des verbes analysés ne comprend qu'un seul changement de relation (et d'emplacement: initial ou final). Le procès de déplacement correspondant à la phrase dans son ensemble peut parfois référer à un second changement de relation (de polarité opposée) mais celui-ci est introduit, de façon compositionnelle, par d'autres éléments et, en particulier, à travers l'utilisation de GP spatiaux et/ou de temps à valeur perfective. ${ }^{15} \mathrm{Ce}$ second changement de relation vient alors compléter, au niveau phrastique, celui directement dénoté par le verbe (Aurnague, à paraître).

Nous terminons cette section en tentant de définir plus précisément la notion de «trajet». Les données recueillies (en particulier la combinaison à par) semblent indiquer qu'un verbe dénote un trajet s'il introduit, au minimum, un changement de relation et un changement d'emplacement. Ces deux changements peuvent être concomitants (si le changement de relation implique un changement d'emplacement) ou successifs (changement d'emplacement subséquent ou antérieur au changement de relation). Les informations spatiales véhiculées par les verbes pourraient théoriquement être plus complexes mais, conformément à ce qui a été dit plus haut, il est peu fréquent qu'un prédicat intransitif (de changement de relation et d'emplacement) intègre, dans son contenu sémantique, un double changement de relation et d'emplacement (initial et final). Comme on l'a indiqué, un changement de relation et d'emplacement de polarité opposée à celui dénoté par le sémantisme verbal peut cependant être introduit au niveau phrastique au moyen, par exemple d'un GP spatial et/ou d'un temps approprié(s). Mais ceci n'est pas systématique et le trajet « total » construit dans le cadre de la phrase peut très bien se 
limiter à un changement de relation et d'emplacement initial ou final et à un site de même polarité : Max est parti de chez lui à 8 heures; Max est arrivé à l'université à 10 heures.

\section{Conclusions}

La notion de changement de relation locative élémentaire, associée à celle de changement d'emplacement, permet de circonscrire les procès de déplacement au sens strict - souvent qualifiés de déplacements téliques ou de changements de lieu - sans recourir à une caractérisation (uniquement) aspectuelle ou à des concepts spatiaux (lieu) qui seraient incompatibles avec d'autres propriétés ontologiques des descriptions spatiales (espace statique) (sections 1 et 2). L'interaction entre ces deux notions a d'abord été étudiée en mettant au jour les particularités des prédicats de changement d'emplacement susceptibles de s'associer à un changement de relation locative élémentaire (concept de tendancialité ; section 3.1). Il a ensuite été montré que les verbes intransitifs de changement de relation et d'emplacement peuvent être distingués par le fait qu'ils comportent un ou deux changements de relation (accompagnés, parfois, d'un changement d'emplacement concomitant ou «conjoint») et que ceux-ci sont précédés ou, plus rarement, suivis d'un changement d'emplacement (section 3.2). Nous avons pris soin de déterminer le statut de ces changements d'emplacement - antérieurs ou subséquents - selon qu'ils sont pleinement intégrés au sémantisme du verbe (et peuvent être dénotés par celui-ci), qu'ils sont simplement présupposés dans le contenu sémantique ou qu'ils n'y sont pas encodés.

Ce travail présente divers types de conséquences et de prolongements possibles (Aurnague, à paraître). Sur le plan de l'expression (inter)linguistique de l'espace, il serait, par exemple, intéressant de vérifier si le fait que certains changements de relation locative élémentaire n'impliquent pas de changement d'emplacement (ex : relation de support/contact) est corroboré par les données d'autres langues (de type «verb-framed ») que le français. Du point de vue de l'interface syntaxe-sémantique, il nous semble que la structure argumentale des verbes de déplacement devrait rendre compte du fait que peu d'entre eux sélectionnent véritablement deux sites. La modélisation syntactico-sémantique devrait, par ailleurs, saisir la manière dont d'autres éléments de la phrase parviennent à introduire un second changement de relation ainsi que la possibilité que ce second changement s'associe à l'éventualité (aux éventualités) initialement dénotée(s) par le verbe ou constitue une éventualité de changement de relation et d'emplacement indépendante. Enfin, nous soutenons que les propriétés relevant de l'aspect interne des verbes doivent pouvoir être déduites de leurs propriétés spatiales, et non l'inverse.

\section{Références bibliographiques}

Asher, N. \& Sablayrolles, P. (1995). A typology and discourse semantics for motion verbs and spatial PPs in French. Journal of Semantics, 12-2:163-209.

Aurnague, M. (1996). Les Noms de Localisation Interne : tentative de caractérisation sémantique à partir de données du basque et du français. Cahiers de Lexicologie, 69, 1996-2 : 159-192.

Aurnague, M. (2000). Entrer par la petite porte, passer par des chemins de traverse : à propos de la préposition par et de la notion de trajet. Carnets de Grammaire, 7. Toulouse : rapport ERSS.

Aurnague, M. (2004). Les structures de l'espace linguistique : regards croisés sur quelques constructions spatiales du basque et du français. Leuven : Peeters.

Aurnague, M. (à paraître). How motion verbs are spatial: the spatial foundations of intransitive motion verbs in French.

Aurnague, M., Champagne, M., Vieu, L., Borillo, A. Muller, P., Nespoulous, J.L. \& Sarda, L. (2007). Categorizing spatial entities with frontal orientation: the role of function, motion and saliency in the processing of the French Internal Localization Nouns avant/devant. In : M. Aurnague, M. Hickmann \& L. Vieu (eds), The categorization of spatial entities in language and cognition. Amsterdam : Benjamins, pp. 153-175.

Aurnague, M. \& Stosic, D. (2002). La préposition par et l'expression du déplacement: vers une caractérisation sémantique et cognitive de la notion de "trajet". Cahiers de Lexicologie, 81, 2002-2 : 113-139. 
Boons, J.P. (1987). La notion sémantique de déplacement dans une classification syntaxique des verbes locatifs. Langue Française, $76: 5-40$.

Boons, J.P., Gillet, A. \& Leclere, C. (1976). La structures des phrases simples en français : constructions intransitives. Genève : Droz.

Borillo, A. (1998). L'espace et son expression en français. Paris : Ophrys.

Bowerman, M., de León, L. \& Choi, S. (1995). Verbs, particles, and spatial semantics: learning to talk about spatial actions in typologically different languages. In : E.V. Clark (ed), Proceedings of the Twenty-Seventh Annual Child Language Research Forum. Stanford, CA : CSLI, pp. 101-110.

Fong, V. \& Poulain, C. (1998). Locating linguistic variation in semantic templates. In : J.P. Koenig (ed), Discourse and cognition: bridging the gap. Stanford, CA : CSLI, pp. 29-39.

Grinevald, C. (à paraître). Directional do it because prepositions don't: path in motion and location in Pop'ti (Mayan). In : H. Cuykens, W. De Mulder, M. Goyens \& T. Mortelmans (eds), Variation and change in adpositions of movement. Amsterdam : Benjamins.

Gross, M. (1975). Méthodes en syntaxe : régime des constructions complétives. Paris : Hermann.

Guillet, A. (1990). Une classification des verbes transitifs locatifs. Thèse de Doctorat d'Etat, Université Paris 7.

Hickmann, M. (2006). The relativity of motion in first language acquisition. In : M. Hickmann \& S. Robert (eds), Space in languages: linguistic systems and cognitive categories. Amsterdam : Benjamins, pp. 281-308.

Jackendoff, R. (1983). Semantic and cognition. Cambridge, MA : MIT Press.

Jackendoff, R. (1990). Semantic structures. Cambridge, MA : MIT Press.

Kamp, H. \& Reyle, U. (1993). From discourse to logic. Dordrecht : Kluwer.

Kopecka, A. (2006). The semantic structures of motion verbs in French. In : M. Hickmann \& S. Robert (eds), Space in languages: linguistic systems and cognitive categories. Amsterdam : Benjamins, pp. 83-101.

Kopecka, A. (à paraître). L'expression du déplacement en français : l'interaction des facteurs sémantiques, aspectuels et pragmatiques dans la construction du sens spatial. A paraître dans Langages (Approches récentes de la préposition, D. Stosic \& W. De Mulder (eds)).

Krifka, M. (1992). Thematic relations as links between nominal reference and temporal constitution. In : I. Sag and A. Szabolsci (eds), Lexical Matters. Stanford, CA : CSLI, pp. 29-53.

Krifka, M. (1995). Telicity in movement. In : P. Amsili, M. Borillo \& L. Vieu (eds), Time, Space and Movement: meaning and knowledge in the sensible world, Working Notes of the $5^{\text {th }}$ International Workshop. Toulouse : LRC, pp. 63-75 (Part A).

Lamiroy, B. (1983). Les verbes de mouvement en français et en espagnol. Amsterdam : Benjamins.

Laur, D. (1991). Sémantique du déplacement et de la localisation en français : une étude des verbes, des prépositions et de leurs relations dans la phrase simple. Thèse de Doctorat, Université de Toulouse-Le Mirail.

Levin, B. (1993). English verb classes and alternations: a preliminary investigation. Chicago : The University of Chicago Press.

Levin, B. \& Rappaport, M. (1992). The lexical semantics of verbs of motion: the perspective from unaccusativity. In : I.M. Roca (ed), The thematic structure: its role in grammar. Berlin : Foris Publications, pp. 247-269.

Levin, B. \& Rappaport, M. (1995). Unaccusativity: at the syntax-lexical semantics interface. Cambridge, MA : MIT Press.

Moens, M. \& Steedman, M. (1988). Temporal ontology and temporal reference. Computational Linguistics, 14-2 : 15-28.

Muller, P. \& Sarda, L. (1998). Représentation de la sémantique des verbes de déplacement transitifs directs du français. TAL, 39-2 : 127-147

Perlmutter, D. (1978). Impersonal passives and the unaccusative hypothesis. BLS, 4 : 157-189. 
Rey-Debove, J. \& Rey, A. (eds) (2007). Le nouveau Petit Robert: dictionnaire alphabétique et analogique de la langue française. Paris: Dictionnaires Le Robert.

Sarda, L. (1999). Contribution à l'étude de la sémantique de l'espace et du temps: analyse des verbes de déplacement transitifs directs du français. Thèse de Doctorat, Université de Toulouse-Le Mirail.

Slobin, D.I. (2003). Language and thought online: cognitive consequences of linguistic relativity. In : D. Gentner \& S. Goldin-Meadow (eds), Language in mind: advances in the investigation of language and thought. Cambridge, MA : MIT Press, pp. 157-191.

Slobin, D.I. (2004). The many ways to search for a frog: linguistic typology and the expression of motion events. In : S. Strömqvist \& L. Verhoeven (eds), Relating events in narrative: typological and contextual perspectives. Mahwah, NJ : Lawrence Erlbaum, pp. 219-257.

Stosic, D. (2002). Par et à travers dans l'expression des relations spatiales : comparaison entre le français et le serbo-croate. Thèse de Doctorat, Université de Toulouse-Le Mirail.

Stosic, D. (2007). The prepositions par and à travers and the categorization of spatial entities in French. In : M. Aurnague, M. Hickmann \& L. Vieu (eds), The categorization of spatial entities in language and cognition. Amsterdam : Benjamins, pp. 71-91.

Talmy, L. (1985). Lexicalization patterns: semantic structure in lexical forms. In : T. Shopen (ed), Language typology and syntactic description (vol. 3): grammatical categories and the lexicon. Cambridge : Cambridge University Press, pp. 57-143.

Talmy, L. (2000). Toward a cognitive semantics (vol. 1 \& 2). Cambridge, MA : MIT Press.

Tenny, C. (1995). How motion verbs are special: the interaction of linguistic and pragmatic information in aspectual verb meanings. Pragmatics and Cognition, 3-1:31-73.

Vandeloise, C. (1986). L'espace en français : sémantique des prépositions spatiales. Paris : Seuil.

Vandeloise, C. (1988). Les usages statiques de la préposition à. Cahiers de Lexicologie, 53 : 119-148.

Vandeloise, C. (2001). Aristote et le lexique de l'espace : rencontres entre la physique grecque et la linguistique cognitive. Stanford, CA : CSLI.

Vendler, Z. (1957). Verbs and times. Philosophical Review, 66 : 143-160.

Vet, C. (1994). Petite grammaire de l'Aktionsart et de l'aspect. Cahiers de Grammaire, 19 : 1-18.

Vetters, C. (1996). Temps, aspect et narration. Amsterdam : Rodopi.

Vieu, L. (1991). Sémantique des relations spatiales et inférences spatio-temporelles : une contribution à l'étude des structures formelles de l'espace en langage naturel. Thèse de Doctorat, Université Paul Sabatier, Toulouse.

\footnotetext{
${ }^{1}$ Nous incluons, ici, sous le label « verbes intransitifs », les prédicats que certaines analyses et grammaires qualifient de transitifs « indirects ».

${ }^{2} \mathrm{~S}$ 'il est fort rare et littéraire aujourd'hui, cet emploi de par était courant au $19^{\mathrm{e}}$ siècle. De nombreuses occurrences sont encore présentes dans les textes de la première moitié du $20^{\mathrm{e}}$ siècle (corpus Frantext) : Après avoir erré par les rues... (Mon corps et moi, R. Crevel, 1925) ; Au crépuscule... la reine de théâtre et Mr Godeau étaient allés se promener par la ville (Mr Godeau intime, M. Jouhandeau, 1926).

${ }^{3}$ Les propriétés fonctionnelles sous-tendant les principales prépositions spatiales impliquent que leur contenu sémantique soit modélisé de façon véritablement «relationnelle» (relation entre cible et site) et non au moyen de simples fonctions s'appliquant à l'entité-site.

${ }^{4}$ Certaines descriptions intégrant des prépositions orientationnelles peuvent aussi impliquer la négation et l'affirmation d'une relation locative élémentaire sans qu'un déplacement soit nécessairement présent (emploi contextuel de devant dans lequel la cible oriente le site ; devant = face à) : Max s'est placé devant l'arbre.

${ }^{5}$ Dans la suite, et sauf indication contraire, le terme de « changement de relation » fait référence à un changement de relation locative élémentaire. C'est notamment le cas lorsqu'un changement de relation locative élémentaire est associé à un changement d'emplacement (« changement de relation et d'emplacement »).
} 


\begin{abstract}
${ }^{6}$ Un contraste similaire à (9-10) apparaît dans les phrases suivantes (mettant en jeu un placard accessible depuis deux pièces différentes) : ?(?)Le papillon s'est posé sur le placard par la chambre vs. Le papillon est entré dans le placard par la chambre.

${ }^{7}$ Le fait que des prédicats tels que monter ou descendre aient pu être utilisés, jusqu'au $19^{\mathrm{e}}$ siècle, avec l'auxiliaire avoir (à côté de l'auxiliaire être) pour dénoter des changements d'emplacement comme des changements de relation et d'emplacement est révélateur, selon nous, de leur appartenance initiale à la classe des changements d'emplacement : Le tas des ouvriers a monté dans la rue, et ces maudits s'en vont... (Le Forgeron, A. Rimbaud, 1870); Monsieur votre argentier a descendu comme un chat le long des murs... (Maître Cornelius, H. de Balzac, 1846); ...il a monté jusqu'au gros chêne (Les maîtres sonneurs, G. Sand, 1865); ...la peinture de son visage a descendu sur la pourpre (La tentation de Saint-Antoine, G. Flaubert, 1849). S'il n'est pas exclusif des changements d'emplacement, le recours à avoir est, en effet, caractéristique de cette catégorie de verbes (Aurnague, à paraître, Lamiroy, 1983, Levin \& Rappaport, 1992, 1995).

${ }^{8}$ Dans les emplois non intentionnels de dégringoler et dévaler, le concept d'entraînement par une force est également présent.

9 Les groupes de verbes ci-dessous figurent parmi les (possibles) prédicats de changement de relation et d'emplacement qui n'ont pas été pris en compte : dérocher, dévisser; jaillir, sourdre; s'approcher, s'éloigner; s'écarter, se pousser; bifurquer, se déporter, se dévier, obliquer. Cependant, plusieurs de ces verbes sont susceptibles d'être analysés dans le cadre conceptuel et théorique utilisé ici et pourraient assez aisément venir compléter la catégorisation présentée dans cette section.
\end{abstract}

${ }^{10}$ Le symbole '...๖' utilisé ici et dans la suite n'a rien à voir avec l'implication ou la conséquence logique. Il signale simplement la transition d'un état (relation locative élémentaire) à un autre (s1 $\ldots \triangleright s 2)$. Cette transition est un événement (e) dont les relations avec les états correspondants peuvent être représentées formellement de la manière suivante (la relation d' « abutment» $\supset \subset$ indique la précédence temporelle immédiate (Kamp \& Reyle, 1993)) : s1 $\supset \subset$ $\mathrm{e} \supset \subset \mathrm{s} 2$.

${ }^{11}$ Les structures parallèles dont la subordonnée est antéposée (ex : Lorsque la pluie s'est mise à tomber, Max partait à l'université) pourraient également être exploitées dans ce genre de test. Il en va de même des constructions dans lesquelles le déplacement imperfectif est exprimé par la proposition subordonnée plutôt que par la principale : $L a$ pluie s'est mise à tomber lorsque/alors que Max partait à l'université.

${ }^{12}$ Selon la nature du verbe et du groupe prépositionnel utilisés, la négation du déplacement subséquent peut s'avérer encore plus problématique : ??Pollux s'est échappé dans la forêt mais il n'y est jamais arrivé. Je remercie A. Kopecka pour ses remarques sur ce dernier point.

${ }^{13}$ Le crochet ouvert signale que le changement d'emplacement intégré dans le sémantisme verbal n'est pas précédé d'un changement de relation qui en constituerait la borne initiale.

${ }^{14}$ Une définition formelle provisoire du contenu sémantique de ces verbes pourrait avoir la forme suivante ( $\supset$ est la précédence temporelle immédiate ; c: cible, s: site $): \mathrm{V}\left(\mathrm{e}^{\prime}, \mathrm{c}, \mathrm{s}\right) \equiv_{\text {déf }} \exists \mathrm{e}$ ch-rel-empl(e',c,s) $\wedge$ ch-empl(e,c) $\wedge \supset\left(\mathrm{e}, \mathrm{e}^{\prime}\right)$.

${ }^{15}$ Noter que, quel que soit le matériau linguistique additionnel, les changements de relation (et d'emplacement) initiaux indépendants ne réfèrent jamais à un changement de relation final. Comme souligné auparavant, l'ajout d'un GP final a pour effet d'introduire une éventualité de déplacement qui doit être distinguée de celle dénotée par le verbe. 